\title{
ON WEAK SEQUENTIAL COMPLETENESS IN BIPROJECTIVE TENSOR PRODUCT SPACES
}

\author{
LEONIDAS N. TSITSAS
}

\begin{abstract}
In this article we are dealing with a study of the weak sequential completeness of $E \hat{\otimes}_{\varepsilon} F$, the completed $\varepsilon$-tensor product of suitable locally convex spaces $E$ and $F$. In particular, certain results of Lewis [3] are extended.
\end{abstract}

1. Introduction. Let $E$ and $F$ be complete locally convex spaces, $E \hat{\otimes}_{\varepsilon} F$ the respective completed (biprojective) $\varepsilon$-tensor product space and let $T$ be the (vector) subspace of $\left(E^{\prime} \otimes F^{\prime}\right)^{*}$, consisting of the $\sigma\left(\left(E^{\prime} \otimes F^{\prime}\right)^{*}, E^{\prime} \otimes F^{\prime}\right)$ limits of all weakly Cauchy sequences in $E \hat{\otimes}_{\varepsilon} F$. In this note it is proved that $E \hat{\otimes}_{\varepsilon} F$ is weakly sequentially complete if, and only if, $E$ and $F$ are weakly sequentially complete and, moreover, every linear map $u \in T$ transfers equicontinuous subsets of $E^{\prime}$ into relatively compact subsets of $F$ (cf. Theorem 3.2 below). From this result a number of corollaries, referred to the weak sequential completeness of the spaces $E \hat{\otimes}_{\varepsilon} F, \varrho_{c}(E, F), \varrho_{b}(E, F)$ and $\varrho_{e}\left(E_{\tau}^{\prime}, F\right)$, are derived. They have a special bearing, into the case under consideration, on certain results of $[3, \S 2]$, formulated for Banach spaces (with the metric approximation property), which also motivated the present work.

2. Notations and preliminaries. All vector spaces considered in the following are over the field $\mathbf{C}$ of complex numbers. The topological spaces involved are assumed to be Hausdorff. For a dual pair $\langle X, Y\rangle$ of vector spaces we denote by $\sigma(X, Y)$ and $\tau(X, Y)$ the weak and the Mackey topology of $X$ respectively. The locally convex spaces thus obtained are denoted by $X_{\sigma}$ and $X_{\tau}$. If $E$ is a locally convex space, we denote by $E^{*}$ and $E^{\prime}$ the algebraic and the topological dual of $E$ respectively. Moreover, we consider the topology $c\left(E^{\prime}, E\right)$ of the absolutely convex compact convergence in $E$ and denote by $E_{c}^{\prime}$ the respectively locally convex space. For two locally convex spaces $E$ and $F$, we denote by $L(E, F)$ and $E(E, F)$ the (vector) space of linear maps and continuous linear maps from $E$ into $F$ respectively. $\varrho_{c}(E, F)\left(\operatorname{resp} . \varrho_{b}(E, F)\right)$ is the space $\mathcal{L}(E, F)$, equipped with the topology of absolutely convex compact (resp. bounded) convergence in $E$. On the other hand, we consider the (vector) space $K\left(E^{\prime}, F\right)$ of all linear maps from $E^{\prime}$ into $F$, which transfers

Received by the editors February 20, 1976 and, in revised form, March 12, 1976. AMS (MOS) subject classifications (1970). Primary 46M05, 46A30; Secondary 47B05.

Key words and phrases. $\varepsilon$-tensor products, weak sequential completeness, compact operators. 
equicontinuous subsets of $E^{\prime}$ into relatively compact subsets of $F$ and denote by $\mathcal{K}\left(E^{\prime}, F\right)$ its (vector) subspace of all weakly $\left(\sigma\left(E^{\prime}, E\right), \sigma\left(F, F^{\prime}\right)\right)$ continuous linear maps. Now we say that a Banach space $E$ has the metric (s)-approximation property if, for all $\varepsilon>0$ and $y_{1}, \ldots, y_{m} \in E$, there is a finite rank operator $u$ on $E$ with $\|u\| \leqslant 1$ and $\left\|u\left(y_{i}\right)-y_{i}\right\| \leqslant \varepsilon$ for all $i=$ $1, \ldots, m[6$, p. 9$]$.

On the other hand, we need for the sake of references in the sequel the following result of [8] which extends it [3, Lemmas 2.1 and 2.2].

LemMA 2.1. Let $\langle X, Y\rangle$ be a dual pair of vector spaces and let $\subseteq$ be a saturated cover of $Y$, consisting of $\sigma(Y, X)$-bounded and $\sigma\left(X^{*}, X\right)$-closed subsets of $Y$. We also consider the locally convex topology $e$ of 5 -convergence on $X, X^{\prime}$ the topological dual of $(X, e)$, the completion $\hat{X}:=(\hat{X}, \hat{e})$ of $(X, e)$ and $a$ (topological vector) subspace $H$ of $\hat{X}$ with $X \subseteq H \subseteq \hat{X}$. Then the topologies $\sigma(H, Y)$ and $\sigma\left(H, X^{\prime}\right)$ have the same $\hat{e}$-bounded null sequences (and hence also the same $\hat{e}$-bounded Cauchy sequences) in $H$.

3. Weak sequential completeness in biprojective tensor products. Let $E$ and $F$ be locally convex spaces and let $\mathcal{L}\left(E_{\tau}^{\prime}, F\right)$ be the (vector) space of continuous linear maps of $E_{\tau}^{\prime}$ into $F$, which, of course, coincides with the (vector) space $\mathcal{L}\left(E_{\sigma}^{\prime}, F_{\sigma}\right)$ of continuous linear maps of $E_{\sigma}^{\prime}$ into $F_{\sigma}$ (cf. [7, p. 429, Proposition 42.2]). Furthermore, we denote by $\mathcal{L}_{e}\left(E_{\tau}^{\prime}, F\right)$ the space $\mathcal{L}\left(E_{\tau}^{\prime}, F\right)$, equipped with the topology of equicontinuous convergence in $E^{\prime}$ and consider the (topological vector) subspace $\varrho_{e}\left(E_{c}^{\prime}, F\right)$ of it, where $\mathcal{L}\left(E_{c}^{\prime}, F\right)$ is the (vector) space of continuous linear maps from $E_{c}^{\prime}$ into $F$. By [5, p. 35, Proposition 5], $\mathcal{L}\left(E_{c}^{\prime}, F\right)=\mathscr{K}\left(E^{\prime}, F\right)(\S 2)$. For a map $u \in L(E, F)$ we denote by ' $u$ its transpose. If $u \in \mathcal{L}\left(E_{\tau}^{\prime}, F\right)$, then ${ }^{t} u \in \mathcal{L}\left(F_{\tau}^{\prime}, E\right)$. Now let $E \otimes F$ be the tensor product (vector) space of $E$ and $F$. Then the respective biprojective (locally convex) topology is the topology $\varepsilon$ of $\subseteq$-convergence on $E \otimes F$, where $\subseteq$ is the family of the sets $A^{\prime} \otimes B^{\prime}$ with $A^{\prime}$ and $B^{\prime}$ weakly closed equicontinuous subsets of $E^{\prime}$ and $F^{\prime}$ respectively. The locally convex space $E \otimes_{\varepsilon} F$ thus obtained is referred to as the (respective) biprojective tensor product space. We denote by $E \hat{\otimes}_{\varepsilon} F$ the completion of $E \otimes_{\varepsilon} F$ and by $\hat{\varepsilon}$ the topology of $E \hat{\otimes}_{\varepsilon} F . E \hat{\otimes}_{\varepsilon} F$ is contained in $\varrho_{e}\left(E_{c}^{\prime}, F\right)$ whenever the last space is complete. Examples of locally convex spaces $E$ and $F$ with $\varrho_{e}\left(E_{c}^{\prime}, F\right)$ complete are treated in [1] and [2].

Now for any element $u$ of $\mathcal{L}\left(E_{\tau}^{\prime}, F\right)$ and for every $x^{\prime} \in E^{\prime}$ and $y^{\prime} \in F^{\prime}$, one has

$$
\left\langle u, x^{\prime} \otimes y^{\prime}\right\rangle=\left\langle u\left(x^{\prime}\right), y^{\prime}\right\rangle=\left\langle x^{\prime},{ }^{t} u\left(y^{\prime}\right)\right\rangle .
$$

On the other hand, if $\left(u_{n}\right)$ is a (weakly) $\sigma\left(E \hat{\otimes}_{\varepsilon} F,\left(E \hat{\otimes}_{\varepsilon} F\right)^{\prime}\right)$-Cauchy sequence in $E \hat{\otimes}_{\varepsilon} F$, then by Grothendieck's completeness theorem, $\left(u_{n}\right)$ is contained in $\left(E^{\prime} \otimes F^{\prime}\right)^{*}$ and it is also clearly $\sigma\left(\left(E^{\prime} \otimes F^{\prime}\right)^{*}, E^{\prime} \otimes F^{\prime}\right)$ Cauchy, so that, by the completeness of

$$
\left(\left(E^{\prime} \otimes F^{\prime}\right)^{*}, \sigma\left(\left(E^{\prime} \otimes F^{\prime}\right)^{*}, E^{\prime} \otimes F^{\prime}\right)\right),
$$


there exists the $\sigma\left(\left(E^{\prime} \otimes F^{\prime}\right)^{*}, E^{\prime} \otimes F^{\prime}\right)$-limit of $\left(u_{n}\right)$. Thus, let $T$ denote the (vector) subspace of

$$
\left(E^{\prime} \otimes F^{\prime}\right)^{*}=L\left(E^{\prime}, F^{*}\right)
$$

consisting of the $\sigma\left(\left(E^{\prime} \otimes F^{\prime}\right)^{*}, E^{\prime} \otimes F^{\prime}\right)$-limits of all (weakly) $\sigma\left(E \hat{\otimes}_{\varepsilon} F,(E\right.$ $\left.\left.\hat{\otimes}_{\varepsilon} F\right)^{\prime}\right)$-Cauchy sequences in $E \hat{\otimes}_{\varepsilon} F$.

We now need

LEMMA 3.1. If $E$ and $F$ are weakly sequentially complete locally convex spaces with $E \hat{\otimes}_{\varepsilon} F \subseteq \mathcal{L}\left(E_{\tau}^{\prime}, F\right)$, then $T \subseteq \mathcal{L}\left(E_{\tau}^{\prime}, F\right)$.

Proof. Let $u \in T$ and let $\left(u_{n}\right)$ be a $\sigma\left(E \hat{\otimes}_{\varepsilon} F,\left(E \hat{\otimes}_{\varepsilon} F\right)^{\prime}\right)$-Cauchy sequence in $E \hat{\otimes}_{\varepsilon} F$ with $u_{n} \rightarrow u$ in the (weak) topology

$$
\sigma\left(\left(E^{\prime} \otimes F^{\prime}\right)^{*}, E^{\prime} \otimes F^{\prime}\right) .
$$

By hypothesis of weak sequential completeness there exist clearly linear maps $v \in L\left(E^{\prime}, F\right)$ and $w \in L\left(F^{\prime}, E\right)$ such that $v\left(x^{\prime}\right)=\lim u_{n}\left(x^{\prime}\right)$ in $F_{\sigma}$ and $w\left(y^{\prime}\right)=\lim ^{t} u_{n}\left(y^{\prime}\right)$ in $E_{\sigma}$ for every $x^{\prime} \in E^{\prime}$ and $y^{\prime} \in F^{\prime}$. On the other hand, by hypothesis that $E \hat{\otimes}_{\varepsilon} F \subseteq \mathcal{L}\left(E_{\tau}^{\prime}, F\right)$ and relation (3.1) above, one clearly has ${ }^{t} v=w$, that is ${ }^{t} v\left(F^{\prime}\right) \subseteq E$ and, hence, $v$ belongs to $\mathcal{L}\left(E_{\sigma}^{\prime}, F_{\sigma}\right)=\mathcal{L}\left(E_{\tau}^{\prime}, F\right)$. But it obviously follows that $u_{n} \rightarrow v$ in the (weak) topology

$$
\sigma\left(\left(E^{\prime} \otimes F^{\prime}\right)^{*}, E^{\prime} \otimes F^{\prime}\right)
$$

so that $u=v$, which proves the assertion.

We are now in a position to state and prove the main result of this note. That is, we have

TheOREM 3.2. Let $E$ and $F$ be complete locally convex spaces and let $T$ be the (vector) subspace of

$$
\left(E^{\prime} \otimes F^{\prime}\right)^{*}=L\left(E^{\prime}, F^{*}\right)
$$

consisting of the $\sigma\left(\left(E^{\prime} \otimes F^{\prime}\right)^{*}, E^{\prime} \otimes F^{\prime}\right)$-limits of all (weakly) $\sigma\left(E \hat{\otimes}_{\varepsilon} F,(E\right.$ $\left.\left.\hat{\otimes}_{\varepsilon} F\right)^{\prime}\right)$-Cauchy sequences in $E \hat{\otimes}_{\varepsilon} F$. On the other hand, we consider the (vector) space $K\left(E^{\prime}, F\right)$ of all linear maps from $E^{\prime}$ into $F$ which transfers equicontinuous subsets of $E^{\prime}$ into relatively compact subsets of $F$, and the following statements:

(1) (a) $E$ and $F$ are weakly sequentially complete.

(b) $T$ is contained in $K\left(E^{\prime}, F\right)$.

(2) $E \hat{\otimes}_{\varepsilon} F$ is weakly sequentially complete.

Then (2) implies (1). If, moreover, one of the spaces $E, F$ and $E_{c}^{\prime}$ has the approximation property, then (1) implies also (2).

Proof. (1) implies (2). Let $\left(u_{n}\right)$ be a $\sigma\left(E \hat{\otimes}_{\varepsilon} F,\left(E \hat{\otimes}_{\varepsilon} F\right)^{\prime}\right)$-Cauchy sequence in $E \hat{\otimes}_{\varepsilon} F$ and $u \in T$ with $u_{n} \rightarrow u$ in the (weak) topology

$$
\sigma\left(\left(E^{\prime} \otimes F^{\prime}\right)^{*}, E^{\prime} \otimes F^{\prime}\right) .
$$

By hypothesis (1)(a) and Lemma 3.1 above, $u$ belongs to $\mathcal{L}\left(E_{\sigma}^{\prime}, F_{\sigma}\right)$ so that by hypothesis (1)(b) and [5, p. 35, Proposition 5] $u$ belongs also to $\mathcal{L}\left(E_{c}^{\prime}, F\right)$. On 
the other hand, by hypotheses and [1, p. 197, Satz 9], $u$ belongs to $E \hat{\otimes}_{\varepsilon} F$ and $u_{n} \rightarrow u$ in the (weak) topology $\sigma\left(E \hat{\otimes}_{\varepsilon} F,\left(E \hat{\otimes}_{\varepsilon} F\right)^{\prime}\right)$ (Lemma 2.1), and hence $E \hat{\otimes}_{\varepsilon} F$ is weakly sequentially complete. Now (2) implies (1). In fact by standard arguments, given, for instance, in the proof of $[4$, p. 167, §9.1], it follows that (2) implies (1)(a). On the other hand, by the fact that $T=E$ $\hat{\otimes}_{\varepsilon} F$ and the techniques of (1) implies (2) above, (2) clearly implies (1)(b), and the proof is completed.

In particular, the following corollaries have a special bearing on respective results of $[3, \S 2]$.

Corollary 3.3. Let $E$ and $F$ be complete locally convex spaces such that $\mathcal{L}\left(E_{\sigma}^{\prime}, F_{\sigma}\right)=\mathcal{K}\left(E^{\prime}, F\right)$. Suppose, moreover, that one of the spaces $E, F$ and $E_{c}^{\prime}$ has the approximation property. Then $E \hat{\otimes}_{\varepsilon} F\left(=\mathcal{L}_{e}\left(E_{c}^{\prime}, F\right)=\mathcal{L}_{e}\left(E_{\tau}^{\prime}, F\right)\right)$ is weakly sequentially complete if (and only if) both $E$ and $F$ are.

Proof. It follows by [1, p. 197, Satz 9] and Theorem 3.2 above.

By Theorem 3.2 and the corresponding techniques of $[3, p .203$, Theorem 2.1], we clearly get

Corollary 3.4. Let $E$ and $F$ be Banach spaces such that $E$ or $F$ have the metric (s)-approximation property and let $E \hat{\otimes}_{\varepsilon} F$ be weakly sequentially complete. Then $\mathcal{L}\left(E_{\tau}^{\prime}, F\right)=\mathcal{K}\left(E^{\prime}, F\right)$.

On the other hand, by Theorem 3.2 in the foregoing and [2, p. 7, Satz 6] we also get

Corollary 3.5. Let $E$ and $F$ be locally convex spaces such that $E$ is quasi-complete bornological (resp. Montel $(L F)$-space) with the approximation property and $F$ is complete. Then $\varrho_{c}(E, F)=E_{c}^{\prime} \hat{\otimes}_{\varepsilon} F\left(\right.$ resp. $\varrho_{b}(E, F)=E_{b}^{\prime}$ $\hat{\otimes}_{\varepsilon} F$ ) is weakly sequentially complete if (and only if) both $E_{c}^{\prime}$ and $F$ (resp. $E_{b}^{\prime}$ and $F$ ) are.

Now if $E$ and $F$ are complete locally convex spaces with $F$ a semi-Montel space which has the approximation property, then by standard arguments, given, for example, in [7, p. 522, Proposition 50.4], it follows that $E \hat{\otimes}_{\varepsilon} F=$ $\mathcal{L}_{e}\left(E_{\tau}^{\prime}, F\right)$, so that by the foregoing we finally have

Corollary 3.6. Let $E$ and $F$ be complete locally convex spaces such that $F$ is a semi-Montel space which has the approximation property (or, in particular, $F$ is a nuclear space). Then $E \hat{\otimes}_{\varepsilon} F=\mathcal{L}_{e}\left(E_{\tau}^{\prime}, F\right)$ is weakly sequentially complete if (and only if) this is the case for $E$.

\section{REFERENCES}

1. K.-D. Bierstedt, Gewichtete Räume stetiger vektorwertiger Funktionen und das injektive Tensorprodukt. I, J. Reine Angew. Math. 259 (1973), 189-210. MR 47 \#417.

2. K.-D. Bierstedt and R. Meise, Lokalkonvexe Unterräume in topologischen Vektorräumen und das $\varepsilon$-Produkt, Manuscripta Math. 8 (1973), 143-172. MR 48 \#411.

3. D. R. Lewis, Conditional weak compactness in certain inductive tensor products, Math. Ann. 201 (1973), 201-209. MR 48 \#4761. 
4. H. H. Schaefer, Topological vector spaces, Macmillan, New York, 1966. MR 33 \# 1689.

5. L. Schwartz, Théorie des distributions à valeurs vectorielles. I, Ann. Inst. Fourier (Grenoble) 7 (1957), 1-141. MR 21 \#6534.

6. S. Simons, If $E^{\prime}$ has the metric approximation property then so does $\left(E, E^{\prime}\right)$, Math. Ann. 203 (1973), 9-10. MR 49 \#7737.

7. F. Treves, Topological vector spaces, distributions and kernels, Academic Press, New York, 1967. MR 37 \# 726.

8. L. Tsitsas, On weak compactness in biprojective tensor product spaces, Math. Nachr. (to appear).

Mathematical Institute, University of Athens, 57 Solonos, Athens (143), Greece 